\title{
Structural, Spectroscopic (FT-IR, Raman, NMR and UV-Vis.) and Computational Studies on N- phenylpropanamide
}

\section{Nuri ÖZTÜRK ${ }^{1 *}$, Halil GÖKCE², Gökhan ALPASLAN ${ }^{2}$, Yelda BİNGÖL ALPASLAN ${ }^{3}$, Can ALAŞALVAR ${ }^{4}$}

ABSTRACT: Structural, vibrational, magnetic and electronic properties of N-phenylpropanamide were analyzed experimentally and theoretically. The molecular geometry optimization parameters, vibrational wavenumbers, proton and carbon NMR chemical shifts, frontier molecular orbitals and UV-Vis. wavelengths were computed with DFT/B3LYP method at the 6-311+G(d,p) basis set to compare the experimental data obtained from the literature. Calculated harmonic vibrational wavenumber assignments were obtained from the potential energy distribution (PED) analysis. Considering that the $\mathrm{N}-\mathrm{H}^{\cdots} \mathrm{O}$ intermolecular hydrogen bond interaction in crystal packing of $\mathrm{N}$ phenylpropanamide may exist, molecular structure parameters and vibration frequencies of these groups in this interaction were investigated. UV-Vis. electronic absorption parameters, HOMO-LUMO analyses and molecular electrostatic potential (MEP) surface of N-phenylpropanamide were studied to explicate electronic transitions, intramolecular charge transfer and interaction sites in the molecule.

Keywords: N-phenylpropanamide, UV-Vis., NMR, DFT, FT-IR, Raman

\footnotetext{
${ }^{1}$ Nuri ÖZTÜRK (Orcid ID: 0000-0001-8742-0160), Giresun University, Dereli Vocational School, Giresun, Turkey

${ }^{2}$ Halil GÖKCE (Orcid ID: 0000-0003-2258-859X), ${ }^{2}$ Gökhan ALPASLAN (Orcid ID: 0000-0002-7982-3266), Giresun University, Vocational School of Health Services, Giresun, Turkey

${ }^{3}$ Yelda BINGÖL ALPASLAN (Orcid ID: 0000-0002-1629-6016), Giresun University, Faculty of Arts and Sciences, Giresun, Turkey

${ }^{4}$ Can ALAŞALVAR (Orcid ID: 0000-0003-0995-4188), Giresun University, Vocational Schools of Technical Sciences, Giresun, Turkey

*Corresponding author: Nuri ÖZTÜRK, e-mail: nuri.ozturk@giresun.edu.tr

*The article was presented as a poster at the 1st International Technology Sciences and Design Syposium held in Giresun on 27-29 June 2018.
} 


\section{INTRODUCTION}

The phenylamides are highly active class of fungicides controlling plant pathogens. Plant phenylamides are similar with phenylamides in insect toxins (Adreva et al., 2007). Herbicidal activity and experimental structure analyses of the bioactive compound phenylamide derivative $\mathrm{N}$ phenylpropanamide were investigated by (Priyadharsini et al., 2013). They concluded that the efficacy of the N-phenylpropanamide against the weeds had been found to be better than other synthetic chemicals. Klees et al. studied about the alfentanil metabolism to N-phenylpropanamide and noralfentanil determined in human livers (Klees et al., 2005). There are many studies about the different synthesis methods of $\mathrm{N}$ phenylpropanamide in literature (Watanabe et al., 1984; Liu et al., 2011; Na, 2011; Nowrouzi and Jonaghani, 2012; Diab et al., 2017). Watanabe et al. reported that a catalytic system for the synthesis of $\mathrm{N}$-substituted amides in their work on nitro compounds reacted with carboxylic acids. They used nitrobenzene and propionic acid to synthesize of N-phenylpropanamide (Watanabe et al., 1984). As another example, the synthesis of $\mathrm{N}$ phenylpropanamide was performed by the reaction of aniline and acryloyl chloride in dry benzene by Diab et al., 2017.

The quantum chemical calculations have been commonly used to identify the structural parameters, vibrational frequencies (IR and Raman), NMR chemical shifts and electronic features (UV-Vis., HOMO, LUMO and MEP) of molecular systems. In addition, quantum chemical calculations provide strong support for experimental studies. Although it is known that the biological importance of $\mathrm{N}$-phenylpropanamide, detailed structural, spectroscopic and electronic studies are not available in the literature, except for NMR studies. The purpose of this study is to present the experimental and theoretical investigations of structural analysis, vibrational frequencies, proton and carbon-13 NMR chemical shifts and electronic (UV-Vis. and HOMOLUMO) features of $\mathrm{N}$-phenylpropanamide.

\section{MATERIALS AND METHODS}

\section{Computational Methods}

The geometry optimization, vibrational wavenumbers, ${ }^{1} \mathrm{H}$ and ${ }^{13} \mathrm{C}$ NMR chemical shifts (in chloroform), UV-Vis. spectroscopic parameters (in chloroform) and HOMO-LUMO analyses of $\mathrm{N}$ phenylpropanamide were computed using the Gaussian 09W program package (Frisch et al., 2009). The visualizations of calculated results were carried out by GaussView 5.0 program (Dennington et al., 2009). In all computations, we were used DFT/B3LYP method with the 6$311+\mathrm{G}(\mathrm{d}, \mathrm{p})$ basis set (Lee et al., 1988; Becke, 1993).

The vibrational (IR and Raman) wavenumbers were computed based on the optimized structure of compound in the gas phase. The harmonic vibrational wavenumbers obtained by calculating by B3LYP/6-311+G(d,p) level were scaled with 0.9613 (Anderson and Uvdal, 2005). The vibrational band assignments were performed in terms of PED (potential energy distribution) using VEDA 4 program (Jamr'oz, 2004). For ${ }^{1} \mathrm{H}$ and ${ }^{13} \mathrm{C}$ NMR isotropic chemical shifts (in chloroform) and UV-Vis. parameters calculations (in chloroform), the optimized molecular geometry of N-phenylpropanamide was obtained at the B3LYP/6-311+G(d,p) level using IEFPCM method (Miertus et al., 1981). Then, NMR chemical shifts of $\mathrm{N}$-phenylpropanamide were computed at mentioned level, model and solvent with GIAO approach (London, 1937; Ditchfield, 1974; Wolinski et al., 1990). Additionally, UVVis. parameters of the $\mathrm{N}$-phenylpropanamide molecule were calculated using aforementioned computational level, solvent model, solvent and time dependent DFT (TD-DFT) method (Runge and Gross). HOMO and LUMO (the frontier molecular orbitals) analyses were simulated using B3LYP/6-311+G(d,p) level to determine charge transfers and electronic transitions in compound. The molecular electrostatic potential (MEP) map was imitated using the optimized molecular geometry of the N-phenylpropanamide molecule. 


\section{RESULTS AND DISCUSSION}

\section{Molecular Structure Analysis}

Since we do not have experimental data, only the calculated bond lengths and bond angles obtained with the B3LYP/6-311+G(d,p) level in gas phase of the title molecule were listed in Table 1. The optimized molecular structures of compound was given in Figure 1. The carbonyl bond length $(\mathrm{C} 5=\mathrm{O} 1)$ was computed as $1.218 \AA$ $\left(1.227 \AA\right.$ under effect of the $\mathrm{N}-\mathrm{H}^{\cdots} \mathrm{O}$ intermolecular hydrogen bond interaction). The
N2-C3 and N2-C5 bond lengths were computed at $1.413 \AA$ and $1.378 \AA$ as theoretically, respectively. The $\mathrm{C} 4-\mathrm{C} 5$ and $\mathrm{C} 4-\mathrm{C} 8$ bond length in the ethylene group were computed as $1.528 \AA$ and $1.529 \AA$, respectively. The $\mathrm{C}-\mathrm{C}$ bond lengths were calculated at the intervals of 1.390-1.403 $\AA$ in phenyl ring. On the other hand, N-H bond length in amide group was calculated as $1.009 \AA$. The $\mathrm{C}-\mathrm{H}$ bond lengths for ethyl group were calculated at the intervals of 1.091-1.097 $\AA$ while calculated at the interval of 1.079-1.086 $\AA$ in phenyl ring.

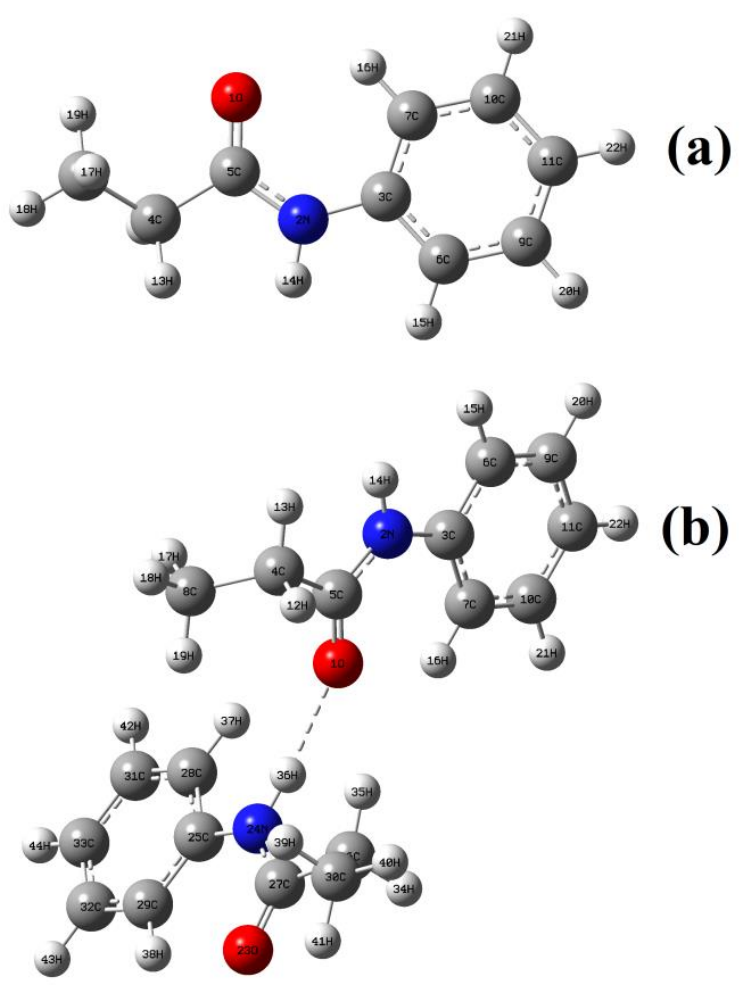

Figure 1. The optimized molecular structures $\left(a=\right.$ for isolated gas phase and $b=$ in presence of $N-H^{\cdots} \mathrm{O}$ interaction) of $\mathrm{N}$-phenylpropanamide.

In the literature, the $\mathrm{C} 5=\mathrm{O} 1, \mathrm{C} 3-\mathrm{N} 2, \mathrm{C} 5$ $\mathrm{N} 2, \mathrm{C} 4-\mathrm{C} 5, \mathrm{C} 4-\mathrm{C} 8$ and $\mathrm{CC}$ (in phenyl ring) bond lengths in 3-(4-hydroxy-3-methoxyphenyl)-Nphenylpropanamide molecule were reported as $1.236(2), 1.420(3), 1.340(2), 1.512(3), 1.534(3)$ and 1.371(3)-1.390(3) $\AA$, respectively (Mierina et al., 2016).

The angles containing the carbonyl group, O1-C5-C4 and O1-C5-N2 were calculated as $122.2^{\circ}$ and $123.8^{\circ}$, respectively. The reason of the Coulombic forces between the ethyl and phenyl groups, the C3-N2-C5 bond angle increased to $129.5^{\circ}$. The $\mathrm{N} 2-\mathrm{C} 5-\mathrm{C} 4$ bond angle was calculated as $114.0^{\circ}$ while $\mathrm{N} 2-\mathrm{C} 3-\mathrm{C} 6$ and $\mathrm{N} 2-\mathrm{C} 3-\mathrm{C} 7$ bond angles were calculated as $117.2^{\circ}$ and $123.4^{\circ}$, respectively. Additionally, the amide tail bond angle $\mathrm{C} 5-\mathrm{C} 4-\mathrm{C} 8$ was calculated as $112.5^{\circ}$ while $\mathrm{C}-\mathrm{C}-\mathrm{C}$ bond angles were computed at the interval of $119.1^{\circ}-121.4^{\circ}$ 
in phenyl ring. As a result of optimization, the title molecule has deviation from plane. The C7C3-N2-C5 and C2-N2-C5-C4 dihedral angles were calculated as $1.2^{\circ}$ and $175.9^{\circ}$, whereas the $\mathrm{N} 2-\mathrm{C} 5-\mathrm{C} 4-\mathrm{C} 8$ torsion angle was computed as $148.6^{\circ}$. Similarly, in the literature, the C3-N2C5, O1-C5-N2, O1-C5-C4, C5-C4-C8, C6-C3$\mathrm{N} 2$ and N2-C5-C4 bond angles in 3-(4-hydroxy3-methoxyphenyl)-N-phenylpropanamide molecule were recorded as $126.8(2)^{\circ}, 121.9(2)^{\circ}$, $121.7(2)^{\circ}, 115.2(2)^{\circ}, 117.8(2)^{\circ}$ and $116.4(2)^{\circ}$, respectively (Mierina et al., 2016).

The $\mathrm{N}-\mathrm{H}^{\cdots} \mathrm{O}$ intermolecular hydrogen bond interaction may exist in crystal packing of the title molecule. In this connection, molecular structure computations for this interaction were performed and its molecular geometry was given in Figure 1(b). The $\mathrm{N}-\mathrm{H}^{\cdots} \mathrm{O}$ intermolecular hydrogen bond parameters were computed 1.016 $\AA$ for $\mathrm{N}-\mathrm{H}$ bond length, $2.020 \AA$ for $\mathrm{H}^{\cdots} \mathrm{O}$ distance, $3.034 \AA$ for $\mathrm{N}^{\cdots} \mathrm{O}$ distance and $175.0^{\circ}$ for $\mathrm{N}-\mathrm{H}^{\cdots} \mathrm{O}$ bond angle.

Table 1. The computed molecular geometry parameters of N-phenylpropanamide.

\begin{tabular}{cccccc}
\hline Bond lengths $(\AA)$ & Calc. & Bond angles $\left(^{\circ}\right)$ & Calc. & Bond angles $\left(^{\circ}\right)$ & Calc. \\
\hline O1-C5 & 1.218 & O1-C5-N2 & 123.8 & C6-C3-C7 & 119.4 \\
N2-C3 & 1.413 & O1-C5-C4 & 122.2 & C6-C9-H20 & 119.5 \\
N2-C5 & 1.378 & N2-C3-C6 & 117.2 & C6-C9-C11 & 120.2 \\
N2-H14 & 1.009 & N2-C3-C7 & 123.4 & C7-C10-C11 & 121.4 \\
C3-C6 & 1.403 & N2-C5-C4 & 114.0 & C7-C10-H21 & 118.8 \\
C3-C7 & 1.401 & C3-N2-C5 & 129.5 & C8-C4-H12 & 109.8 \\
C4-C5 & 1.528 & C3-N2-H14 & 114.7 & C8-C4-H13 & 110.7 \\
C4-C8 & 1.529 & C3-C6-C9 & 120.6 & C9-C6-H15 & 119.8 \\
C4-H12 & 1.097 & C3-C6-H15 & 119.7 & C9-C11-C10 & 119.1 \\
C4-H13 & 1.095 & C3-C7-C10 & 119.3 & C9-C11-H22 & 120.4 \\
C6-C9 & 1.390 & C3-C7-H16 & 119.6 & C10-C7-H16 & 121.0 \\
C6-H15 & 1.086 & C4-C8-H17 & 111.1 & C10-C11-H22 & 120.5 \\
C7-C10 & 1.394 & C4-C8-H18 & 110.5 & C11-C9-H20 & 120.3 \\
C7-H16 & 1.079 & C4-C8-H19 & 110.4 & C11-C10-H21 & 119.9 \\
C8-H17 & 1.093 & C5-C4-C8 & 112.5 & H12-C4-H13 & 106.4 \\
C8-H18 & 1.093 & C5-C4-H12 & 106.5 & H17-C8-H18 & 108.2 \\
C8-H19 & 1.091 & C5-C4-H13 & 110.6 & H17-C8-H19 & 107.8 \\
C9-C11 & 1.394 & C5-N2-H14 & 115.7 & H18-C8-H19 & 108.7 \\
C9-H20 & 1.084 & & & & \\
C10-C11 & 1.393 & 1.084 & & & \\
C10-H21 & 1.084 & & & &
\end{tabular}

\section{Vibrational Frequencies Analysis}

$\mathrm{N}$-phenylpropanamide $\left(\mathrm{C}_{9} \mathrm{H}_{11} \mathrm{NO}\right)$ has 22 atoms and $60(3 \mathrm{~N}-6)$ vibrational modes. The vibrational assignments, experimental frequencies and calculated vibrational wavenumbers, IR intensities and Raman scattering activities of $\mathrm{N}$-phenylpropanamide were listed in Table 2. The vibrational parameters were calculated with the B3LYP/6$311+\mathrm{G}(\mathrm{d}, \mathrm{p})$ level. The simulated and experimental IR (Bio-Rad, 2018) and Raman (AIST, 2018) spectra were given in Figures 2 and 3, respectively. The $\mathrm{NH}$ stretching vibrational mode was observed at $3240 \mathrm{~cm}^{-1}$ in IR and $3295 \mathrm{~cm}^{-1}$ in Raman spectrum while computed at $3477 \mathrm{~cm}^{-1}$ with $100 \%$ contribution of PED (Colthup et al., 1964; Bellamy, 1975; Silverstein and Webster, 1998; Stuart, 2004). The NH stretching mode was computed at 3374 $\mathrm{cm}^{-1}$ due to the $\mathrm{N}-\mathrm{H}^{\cdots} \mathrm{O}$ intermolecular hydrogen bond interaction in crystal packing (Bilkan, 2019). Moreover, NH in-plane bending vibration was observed at 1597, 1548 and $1197 \mathrm{~cm}^{-1}$ in IR and 1557, 1501 and $1205 \mathrm{~cm}^{-1}$ in Raman spectrum of $\mathrm{N}$-phenylpropanamide while computed at 1570, 1494 and $1210 \mathrm{~cm}^{-1}$, Raman 
spectrum of $\mathrm{N}$-phenylpropanamide while computed at 1570, 1494 and $1210 \mathrm{~cm}^{-1}$, respectively. As expected the aromatic ring $\mathrm{CH}$ stretching vibrations were observed at interval of 3178-3027 $\mathrm{cm}^{-1}$ both IR and Raman spectra while calculated in the interval at $3118-3026 \mathrm{~cm}^{-}$ 1 . The observed peaks at 2965 and $2920 \mathrm{~cm}^{-1}$ in the IR spectrum and the peaks in the interval of $2975-2880 \mathrm{~cm}^{-1}$ in the Raman spectrum were assigned to the symmetric and asymmetric $\mathrm{CH}$ stretching modes of ethyl group which were calculated in the interval of $3002-2899 \mathrm{~cm}^{-1}$. The scissoring modes of methylene and methyl groups were found at 1445,1418 and $1370 \mathrm{~cm}^{-1}$ in IR spectrum, 1458, 1445, 1418 and $1374 \mathrm{~cm}^{-1}$ in Raman spectrum and 1448, 1437, 1412, 1407 and $1358 \mathrm{~cm}^{-1}$ with the B3LYP/6-311+G(d,p) level. The experimental peak at 1655 (IR)/1686 (R), calculated at $1685 \mathrm{~cm}^{-1}$ with $80 \%$ contribution of PED, was assigned to significative $\mathrm{C}=\mathrm{O}$ stretching vibration (Colthup et al., 1964; Öztürk et al., 2018; Y1ldırım, 2018). Additionally, the $\mathrm{C}=\mathrm{O}$ stretching vibration was calculated at $1652 \mathrm{~cm}^{-1}$ due to presence of the $\mathrm{N}$ $\mathrm{H}^{\cdots} \mathrm{O}$ intermolecular hydrogen bond interaction in crystal packing (Bilkan, 2017). The CN stretching vibrational mode of $\mathrm{N}$ phenylpropanamide compound was observed at 1548 and $1197 \mathrm{~cm}^{-1}$ in IR spectrum and 1501, 1205 and $1162 \mathrm{~cm}^{-1}$ in Raman spectrum while they were calculated at 1494, 1210 and $1147 \mathrm{~cm}$ ${ }^{1}$ with 15,27 and $25 \%$ contributions of PED, respectively. Aromatic skeletal stretching vibrations observed at $1607 / 1597 \mathrm{~cm}^{-1}$ and $1606 / 1557 \mathrm{~cm}^{-1}$ in the experimental IR/Raman spectrum were computed at $1494 \mathrm{~cm}^{-1}$ and 1467 $\mathrm{cm}^{-1}$. The other stretching, torsion, in-plane and out-of-plane bending stretching vibration modes of the N-phenylpropanamide were listed in Table 2.

Table 2. Vibrational wavenumbers and their assignments of $\mathrm{N}$-phenylpropanamide.

\begin{tabular}{|c|c|c|c|c|c|}
\hline \multirow{2}{*}{ Assignments (PED\%) } & \multicolumn{2}{|c|}{ Exp. $\left(\mathrm{cm}^{-1}\right)$} & \multicolumn{3}{|c|}{ B3LYP/6-311+G(d,p) } \\
\hline & IR & Raman & Calc. & $\mathrm{I}_{\mathrm{IR}}$ & $\mathrm{S}_{\text {Raman }}$ \\
\hline$v(\mathrm{~N} 2-\mathrm{H} 14)(100)$ & 3240 & 3295 & 3477 & 19.32 & 84.71 \\
\hline$v(\mathrm{C} 6-\mathrm{H} 15)(20)+v(\mathrm{C} 7-\mathrm{H} 16)(21)+v(\mathrm{C} 9-\mathrm{H} 20)(29)+v(\mathrm{C} 10-\mathrm{H} 21)(30)$ & 3178 & 3095 & 3118 & 5.28 & 47.58 \\
\hline$v(\mathrm{C} 6-\mathrm{H} 15)(16)+v(\mathrm{C} 10-\mathrm{H} 21)(23)+v(\mathrm{C} 11-\mathrm{H} 22)(58)$ & 3121 & - & 3068 & 16.44 & 264.04 \\
\hline$v(\mathrm{C} 7-\mathrm{H} 16)(35)+v(\mathrm{C} 9-\mathrm{H} 20)(56)$ & 3072 & 3069 & 3054 & 21.47 & 80.83 \\
\hline$v(\mathrm{C} 6-\mathrm{H} 15)(20)+v(\mathrm{C} 10-\mathrm{H} 21)(34)+v(\mathrm{C} 11-\mathrm{H} 22)(36)$ & - & 3054 & 3045 & 0.24 & 92.25 \\
\hline$v(\mathrm{C} 6-\mathrm{H} 15)(43)+v(\mathrm{C} 7-\mathrm{H} 16)(35)+v(\mathrm{C} 9-\mathrm{H} 20)(12)$ & 3027 & - & 3026 & 13.14 & 57.13 \\
\hline$v(\mathrm{C} 8-\mathrm{H} 18)(12)+v(\mathrm{C} 8-\mathrm{H} 19)(79)$ & - & - & 3002 & 14.10 & 41.87 \\
\hline$v(\mathrm{C} 4-\mathrm{H} 13)(62)+v(\mathrm{C} 8-\mathrm{H} 17)(38)$ & 2965 & 2975 & 2980 & 30.34 & 86.45 \\
\hline$v(\mathrm{C} 4-\mathrm{H} 13)(36)+v(\mathrm{C} 8-\mathrm{H} 17)(54)$ & - & - & 2931 & 18.61 & 70.64 \\
\hline$v(\mathrm{C} 4-\mathrm{H} 12)(42)+v(\mathrm{C} 8-\mathrm{H} 18)(36)+v(\mathrm{C} 8-\mathrm{H} 19)(16)$ & 2920 & 2915 & 2921 & 30.60 & 176.35 \\
\hline$v(\mathrm{C} 4-\mathrm{H} 12)(49)+v(\mathrm{C} 8-\mathrm{H} 18)(47)$ & - & 2880 & 2899 & 17.89 & 144.68 \\
\hline$v(\mathrm{O} 1-\mathrm{C} 5)(80)$ & 1655 & 1686 & 1685 & 212.31 & 57.62 \\
\hline$v(\mathrm{C} 6-\mathrm{C} 9)(53)+\beta(\mathrm{H} 15-\mathrm{C} 6-\mathrm{C} 9)(20)$ & 1607 & 1606 & 1579 & 51.37 & 134.46 \\
\hline$v(\mathrm{C} 7-\mathrm{C} 10)(13)+v(\mathrm{C} 9-\mathrm{C} 11)(16)+v(\mathrm{C} 3-\mathrm{C} 7)(24)+\beta(\mathrm{C} 3-\mathrm{C} 7-\mathrm{C} 10)(11)+\beta(\mathrm{H} 14-\mathrm{N} 2-\mathrm{C} 3)(12)$ & 1597 & 1557 & 1570 & 42.55 & 4.49 \\
\hline$v(\mathrm{C} 9-\mathrm{C} 11)(14)+v(\mathrm{~N} 2-\mathrm{C} 5)(15)+\beta(\mathrm{H} 14-\mathrm{N} 2-\mathrm{C} 3)(43)$ & 1548 & 1501 & 1494 & 336.44 & 46.47 \\
\hline$\beta(\mathrm{H} 21-\mathrm{C} 10-\mathrm{C} 11)(56)$ & 1475 & 1482 & 1467 & 61.39 & 10.63 \\
\hline$\beta(\mathrm{H} 13-\mathrm{C} 4-\mathrm{H} 12)(34)+\beta(\mathrm{H} 17-\mathrm{C} 8-\mathrm{H} 19)(17)+\beta(\mathrm{H} 18-\mathrm{C} 8-\mathrm{H} 17)(23)+\tau(\mathrm{H} 19-\mathrm{C} 8-\mathrm{C} 4-\mathrm{C} 5)(11)$ & 1445 & 1458 & 1448 & 12.82 & 5.43 \\
\hline$\beta(\mathrm{H} 17-\mathrm{C} 8-\mathrm{H} 19)(43)+\beta(\mathrm{H} 18-\mathrm{C} 8-\mathrm{H} 17)(32)+\tau(\mathrm{H} 13-\mathrm{C} 4-\mathrm{C} 5-\mathrm{N} 2)(10)$ & - & 1445 & 1437 & 9.22 & 8.98 \\
\hline$\beta(\mathrm{H} 13-\mathrm{C} 4-\mathrm{H} 12)(26)+\beta(\mathrm{H} 17-\mathrm{C} 8-\mathrm{H} 19)(13)+\beta(\mathrm{H} 19-\mathrm{C} 8-\mathrm{H} 18)(22)$ & 1418 & 1418 & 1412 & 29.93 & 7.03 \\
\hline$\beta(\mathrm{H} 16-\mathrm{C} 7-\mathrm{C} 10)(14)+\beta(\mathrm{H} 22-\mathrm{C} 11-\mathrm{C} 10)(16)$ & - & - & 1407 & 103.29 & 14.65 \\
\hline$\beta(\mathrm{H} 13-\mathrm{C} 4-\mathrm{H} 12)(13)+\beta(\mathrm{H} 18-\mathrm{C} 8-\mathrm{H} 17)(11)+\beta(\mathrm{H} 19-\mathrm{C} 8-\mathrm{H} 18)(69)$ & 1370 & 1374 & 1358 & 5.95 & 1.97 \\
\hline$\beta(\mathrm{H} 12-\mathrm{C} 4-\mathrm{C} 8)(15)+\tau(\mathrm{H} 13-\mathrm{C} 4-\mathrm{C} 5-\mathrm{N} 2)(34)$ & 1312 & - & 1324 & 30.77 & 23.07 \\
\hline$v(\mathrm{C} 7-\mathrm{C} 10)(31)+\beta(\mathrm{H} 20-\mathrm{C} 9-\mathrm{C} 11)(45)+\beta(\mathrm{H} 22-\mathrm{C} 11-\mathrm{C} 10)(11)$ & 1304 & 1303 & 1304 & 1.14 & 5.72 \\
\hline$v(\mathrm{C} 7-\mathrm{C} 10)(19)+v(\mathrm{C} 3-\mathrm{C} 7)(27)+v(\mathrm{~N} 2-\mathrm{C} 5)(12)+\beta(\mathrm{H} 20-\mathrm{C} 9-\mathrm{C} 11)(20)$ & 1297 & 1257 & 1282 & 93.05 & 32.75 \\
\hline$\beta(\mathrm{H} 12-\mathrm{C} 4-\mathrm{C} 8)(60)$ & 1248 & - & 1240 & 6.53 & 3.60 \\
\hline$v(\mathrm{~N} 2-\mathrm{C} 5)(27)+\beta(\mathrm{H} 14-\mathrm{N} 2-\mathrm{C} 3)(11)$ & 1197 & 1205 & 1210 & 62.28 & 82.71 \\
\hline$v(\mathrm{C} 6-\mathrm{C} 9)(15)+\beta(\mathrm{H} 15-\mathrm{C} 6-\mathrm{C} 9)(72)$ & - & 1176 & 1158 & 0.22 & 14.31 \\
\hline$v(\mathrm{~N} 2-\mathrm{C} 3)(25)+\tau(\mathrm{H} 13-\mathrm{C} 4-\mathrm{C} 5-\mathrm{N} 2)(10)$ & - & 1162 & 1147 & 119.93 & 3.66 \\
\hline$v(\mathrm{C} 7-\mathrm{C} 10)(16)+\beta(\mathrm{H} 16-\mathrm{C} 7-\mathrm{C} 10)(39)+\beta(\mathrm{H} 22-\mathrm{C} 11-\mathrm{C} 10)(32)$ & - & 1154 & 1137 & 3.10 & 5.16 \\
\hline$v(\mathrm{C} 9-\mathrm{C} 11)(43)+\beta(\mathrm{H} 16-\mathrm{C} 7-\mathrm{C} 10)(23)+\beta(\mathrm{H} 22-\mathrm{C} 11-\mathrm{C} 10)(16)$ & 1069 & 1075 & 1069 & 10.54 & 1.45 \\
\hline
\end{tabular}




\begin{tabular}{|c|c|c|c|c|c|}
\hline$\beta(\mathrm{C} 8-\mathrm{C} 4-\mathrm{C} 5)(11)+\tau(\mathrm{H} 12-\mathrm{C} 4-\mathrm{C} 5-\mathrm{N} 2)(18)+\tau(\mathrm{H} 19-\mathrm{C} 8-\mathrm{C} 4-\mathrm{C} 5)(31)$ & - & 1056 & 1054 & 2.03 & 5.28 \\
\hline$v(\mathrm{C} 8-\mathrm{C} 4)(16)+\tau(\mathrm{H} 17-\mathrm{C} 8-\mathrm{C} 4-\mathrm{C} 5)(34)$ & 1030 & - & 1043 & 13.78 & 4.00 \\
\hline$v(\mathrm{C} 10-\mathrm{C} 11)(38)+\beta(\mathrm{C} 3-\mathrm{C} 7-\mathrm{C} 10)(10)+\beta(\mathrm{H} 21-\mathrm{C} 10-\mathrm{C} 11)(24)$ & 1013 & 1001 & 1010 & 1.86 & 18.80 \\
\hline$v(\mathrm{C} 4-\mathrm{C} 5)(14)+v(\mathrm{C} 8-\mathrm{C} 4)(38)+\beta(\mathrm{C} 6-\mathrm{C} 9-\mathrm{C} 11)(11)+\tau(\mathrm{H} 17-\mathrm{C} 8-\mathrm{C} 4-\mathrm{C} 5)(12)$ & 998 & - & 981 & 2.09 & 2.78 \\
\hline$v(\mathrm{C} 10-\mathrm{C} 11)(34)+\beta(\mathrm{C} 6-\mathrm{C} 9-\mathrm{C} 11)(47)$ & - & - & 972 & 0.68 & 56.96 \\
\hline $\begin{array}{l}\tau(\mathrm{H} 15-\mathrm{C} 6-\mathrm{C} 9-\mathrm{C} 11)(20)+\tau(\mathrm{H} 16-\mathrm{C} 7-\mathrm{C} 10-\mathrm{C} 11)(33)+\tau(\mathrm{H} 22-\mathrm{C} 11-\mathrm{C} 10-\mathrm{C} 7)(10)+\tau(\mathrm{C} 7-\mathrm{C} 10-\mathrm{C} 11- \\
\mathrm{C} 9)(25)\end{array}$ & - & - & 968 & 0.88 & 0.31 \\
\hline$\tau(\mathrm{H} 15-\mathrm{C} 6-\mathrm{C} 9-\mathrm{C} 11)(58)+\tau(\mathrm{H} 22-\mathrm{C} 11-\mathrm{C} 10-\mathrm{C} 7)(17)$ & 926 & 930 & 943 & 0.18 & 0.09 \\
\hline$v(\mathrm{C} 4-\mathrm{C} 5)(13)+v(\mathrm{C} 8-\mathrm{C} 4)(12)+\beta(\mathrm{O} 1-\mathrm{C} 5-\mathrm{N} 2)(20)+\beta(\mathrm{C} 5-\mathrm{N} 2-\mathrm{C} 3)(15)$ & 899 & 900 & 889 & 11.52 & 2.68 \\
\hline$\tau(\mathrm{H} 16-\mathrm{C} 7-\mathrm{C} 10-\mathrm{C} 11)(30)+\tau(\mathrm{H} 20-\mathrm{C} 9-\mathrm{C} 11-\mathrm{C} 10)(23)+\tau(\mathrm{H} 22-\mathrm{C} 11-\mathrm{C} 10-\mathrm{C} 7)(24)$ & - & - & 884 & 6.87 & 0.24 \\
\hline$\tau(\mathrm{H} 21-\mathrm{C} 10-\mathrm{C} 11-\mathrm{C} 9)(76)$ & 835 & 836 & 812 & 0.13 & 1.11 \\
\hline$v(\mathrm{C} 3-\mathrm{C} 7)(11)+v(\mathrm{~N} 2-\mathrm{C} 3)(10)+\beta(\mathrm{C} 6-\mathrm{C} 9-\mathrm{C} 11)(12)+\tau(\mathrm{H} 21-\mathrm{C} 10-\mathrm{C} 11-\mathrm{C} 9)(11)$ & 800 & - & 810 & 0.75 & 5.72 \\
\hline$\tau(\mathrm{H} 18-\mathrm{C} 8-\mathrm{C} 4-\mathrm{C} 5)(34)+\gamma(\mathrm{O} 1-\mathrm{C} 4-\mathrm{N} 2-\mathrm{C} 5)(20)$ & 755 & 763 & 775 & 5.02 & 2.35 \\
\hline $\begin{array}{l}\tau(\mathrm{H} 16-\mathrm{C} 7-\mathrm{C} 10-\mathrm{C} 11)(12)+\tau(\mathrm{H} 20-\mathrm{C} 9-\mathrm{C} 11-\mathrm{C} 10)(24)+\tau(\mathrm{H} 22-\mathrm{C} 11-\mathrm{C} 10-\mathrm{C} 7)(21)+\tau(\mathrm{C} 3-\mathrm{C} 7-\mathrm{C} 10- \\
\mathrm{C} 11)(14)+\gamma(\mathrm{N} 2-\mathrm{C} 6-\mathrm{C} 7-\mathrm{C} 3)(17)\end{array}$ & 750 & - & 739 & 57.85 & 1.87 \\
\hline$v(\mathrm{C} 4-\mathrm{C} 5)(14)+\beta(\mathrm{O} 1-\mathrm{C} 5-\mathrm{N} 2)(11)+\tau(\mathrm{C} 3-\mathrm{C} 7-\mathrm{C} 10-\mathrm{C} 11)(10)+\gamma(\mathrm{O} 1-\mathrm{C} 4-\mathrm{N} 2-\mathrm{C} 5)(14)$ & 695 & 685 & 679 & 11.28 & 3.56 \\
\hline$\tau(\mathrm{H} 20-\mathrm{C} 9-\mathrm{C} 11-\mathrm{C} 10)(13)+\tau(\mathrm{H} 22-\mathrm{C} 11-\mathrm{C} 10-\mathrm{C} 7)(14)+\tau(\mathrm{C} 3-\mathrm{C} 7-\mathrm{C} 10-\mathrm{C} 11)(31)$ & - & - & 678 & 27.72 & 1.19 \\
\hline$\beta(\mathrm{C} 3-\mathrm{C} 7-\mathrm{C} 10)(13)+\beta(\mathrm{C} 7-\mathrm{C} 10-\mathrm{C} 11)(42)+\beta(\mathrm{C} 10-\mathrm{C} 11-\mathrm{C} 9)(33)$ & 604 & 618 & 608 & 0.70 & 4.45 \\
\hline$v(\mathrm{C} 4-\mathrm{C} 5)(11)+\beta(\mathrm{C} 10-\mathrm{C} 11-\mathrm{C} 9)(15)+\gamma(\mathrm{O} 1-\mathrm{C} 4-\mathrm{N} 2-\mathrm{C} 5)(23)$ & 576 & - & 601 & 2.49 & 3.35 \\
\hline$\beta(\mathrm{C} 10-\mathrm{C} 11-\mathrm{C} 9)(10)+\tau(\mathrm{H} 14-\mathrm{N} 2-\mathrm{C} 3-\mathrm{C} 6)(44)$ & - & 570 & 550 & 27.78 & 1.13 \\
\hline$\tau(\mathrm{H} 14-\mathrm{N} 2-\mathrm{C} 3-\mathrm{C} 6)(44)$ & - & 510 & 517 & 16.78 & 1.48 \\
\hline$\tau(\mathrm{H} 20-\mathrm{C} 9-\mathrm{C} 11-\mathrm{C} 10)(17)+\tau(\mathrm{C} 7-\mathrm{C} 10-\mathrm{C} 11-\mathrm{C} 9)(17)+\gamma(\mathrm{N} 2-\mathrm{C} 6-\mathrm{C} 7-\mathrm{C} 3)(34)$ & 499 & - & 493 & 25.77 & 0.23 \\
\hline$\tau(\mathrm{H} 15-\mathrm{C} 6-\mathrm{C} 9-\mathrm{C} 11)(16)+\tau(\mathrm{C} 6-\mathrm{C} 9-\mathrm{C} 11-\mathrm{C} 10)(82)$ & - & - & 402 & 0.06 & 0.02 \\
\hline$\beta(\mathrm{O} 1-\mathrm{C} 5-\mathrm{N} 2)(30)+\beta(\mathrm{C} 5-\mathrm{N} 2-\mathrm{C} 3)(40)$ & - & 390 & 344 & 4.91 & 1.51 \\
\hline$\nu(\mathrm{N} 2-\mathrm{C} 3)(27)+\beta(\mathrm{C} 10-\mathrm{C} 11-\mathrm{C} 9)(10)+\beta(\mathrm{C} 4-\mathrm{C} 5-\mathrm{N} 2)(19)$ & - & 328 & 300 & 0.66 & 5.00 \\
\hline$\beta(\mathrm{N} 2-\mathrm{C} 3-\mathrm{C} 7)(16)+\beta(\mathrm{C} 4-\mathrm{C} 5-\mathrm{N} 2)(15)+\beta(\mathrm{C} 8-\mathrm{C} 4-\mathrm{C} 5)(30)$ & - & 270 & 280 & 8.61 & 0.51 \\
\hline$\beta(\mathrm{N} 2-\mathrm{C} 3-\mathrm{C} 7)(11)+\tau(\mathrm{C} 7-\mathrm{C} 10-\mathrm{C} 11-\mathrm{C} 9)(23)+\tau(\mathrm{C} 3-\mathrm{C} 7-\mathrm{C} 10-\mathrm{C} 11)(14)+\tau(\mathrm{C} 4-\mathrm{C} 5-\mathrm{N} 2-\mathrm{C} 3)(13)$ & - & - & 242 & 3.02 & 0.79 \\
\hline$\tau(\mathrm{H} 12-\mathrm{C} 4-\mathrm{C} 5-\mathrm{N} 2)(38)+\tau(\mathrm{H} 18-\mathrm{C} 8-\mathrm{C} 4-\mathrm{C} 5)(22)+\tau(\mathrm{H} 19-\mathrm{C} 8-\mathrm{C} 4-\mathrm{C} 5)(14)$ & - & - & 201 & 1.47 & 0.26 \\
\hline$\beta(\mathrm{N} 2-\mathrm{C} 3-\mathrm{C} 7)(55)+\beta(\mathrm{C} 4-\mathrm{C} 5-\mathrm{N} 2)(24)$ & - & 115 & 133 & 1.93 & 0.34 \\
\hline$\tau(\mathrm{C} 4-\mathrm{C} 5-\mathrm{N} 2-\mathrm{C} 3)(57)+\gamma(\mathrm{N} 2-\mathrm{C} 6-\mathrm{C} 7-\mathrm{C} 3)(13)$ & - & - & 78 & 0.22 & 1.74 \\
\hline$\tau(\mathrm{C} 5-\mathrm{N} 2-\mathrm{C} 3-\mathrm{C} 6)(81)$ & - & - & 49 & 5.35 & 0.54 \\
\hline$\tau(\mathrm{H} 12-\mathrm{C} 4-\mathrm{C} 5-\mathrm{N} 2)(10)+\tau(\mathrm{C} 4-\mathrm{C} 5-\mathrm{N} 2-\mathrm{C} 3)(12)+\tau(\mathrm{C} 8-\mathrm{C} 4-\mathrm{C} 5-\mathrm{N} 2)(64)$ & - & - & 30 & 0.15 & 0.85 \\
\hline
\end{tabular}

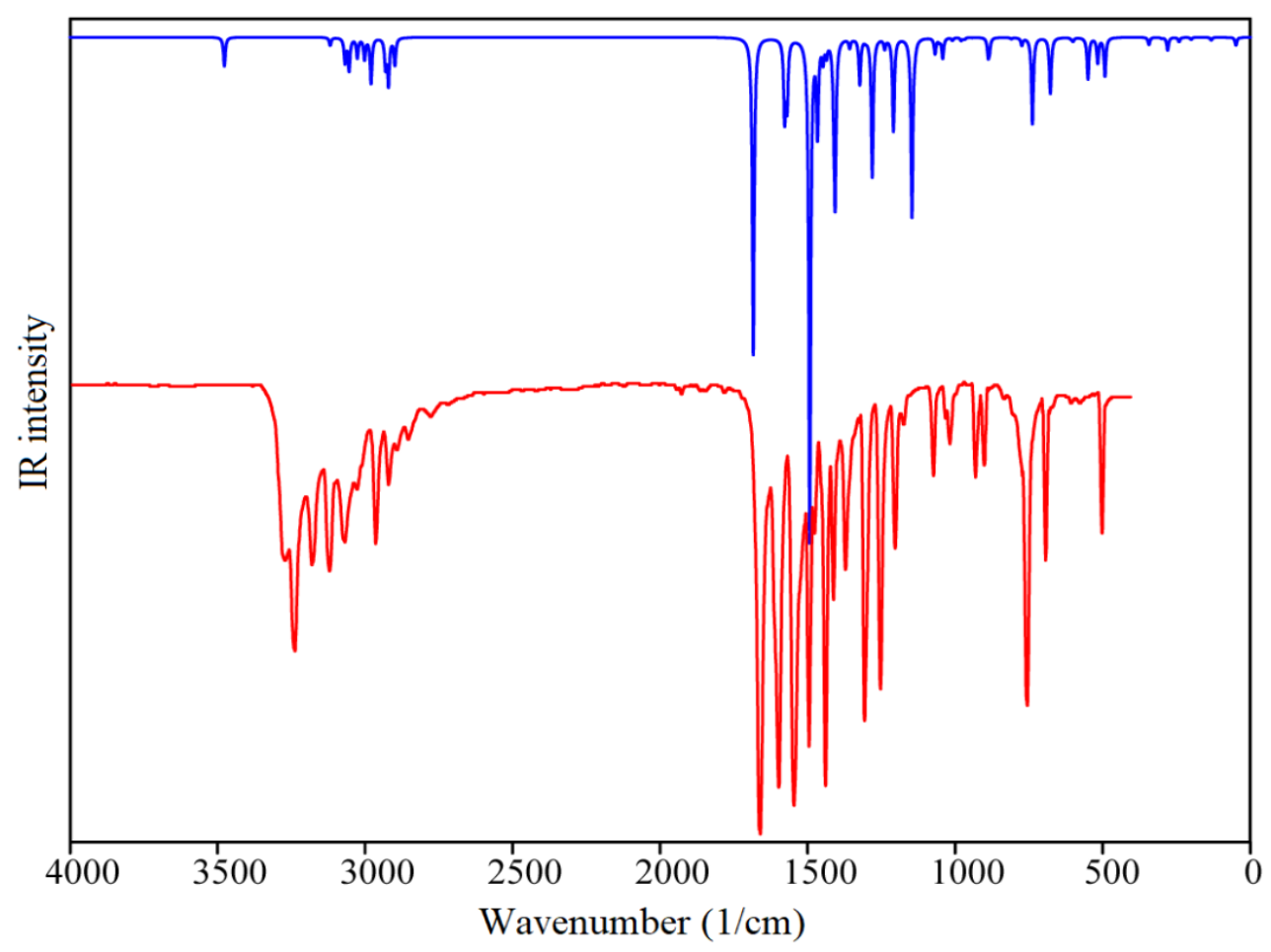

Figure 2. The sim. (top) and exp. (bottom) IR spectra of N-phenylpropanamide. 


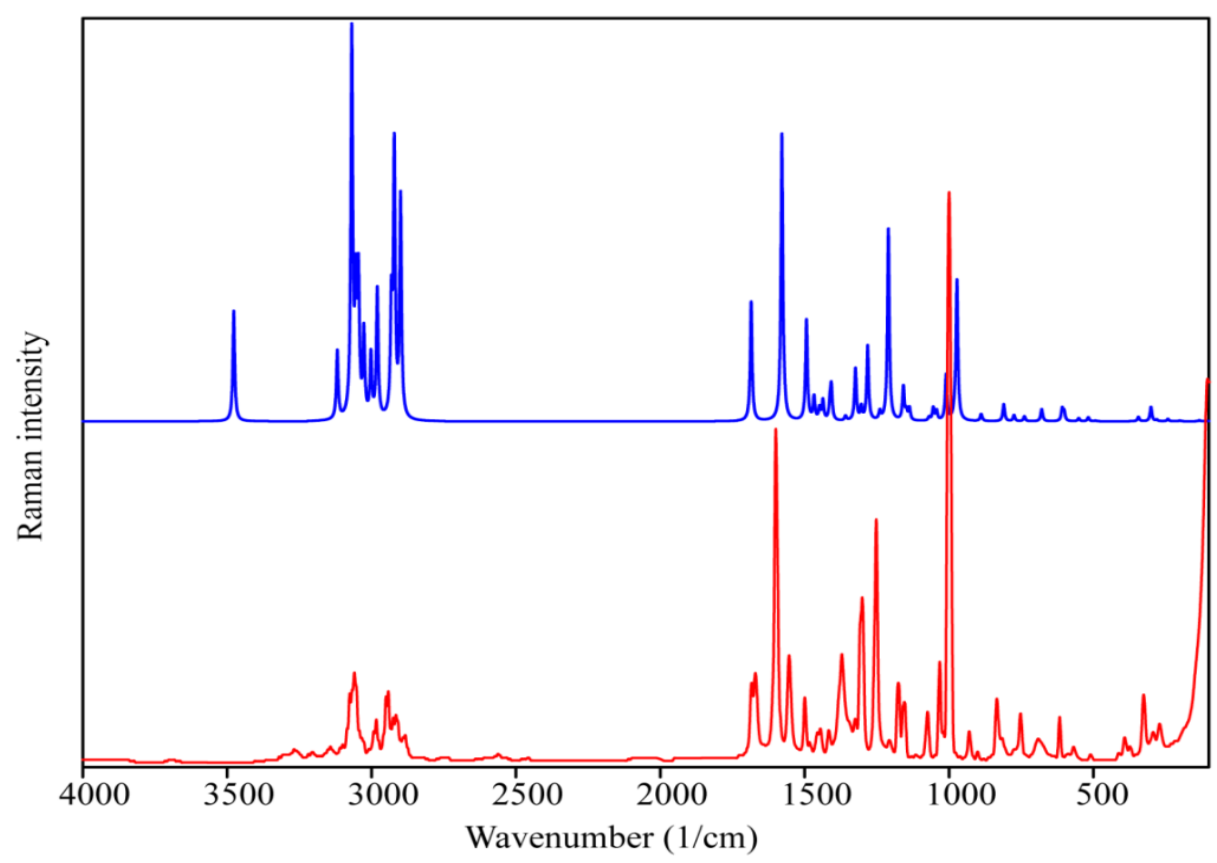

Figure 3. The sim. (top) and exp. (bottom) Raman spectra of N-phenylpropanamide.

\section{NMR Chemical Shift Analysis}

The experimental ${ }^{1} \mathrm{H}$ and ${ }^{13} \mathrm{C} \quad \mathrm{NMR}$ isotropic chemical shift values recorded in $\mathrm{CDCl}_{3}$ were taken from (AIST, 2018) and (Watanabe et al., 1984). In order to make a comparison of experimental values and calculated data, NMR chemical shifts calculated via GIAO method with the B3LYP/6$311+\mathrm{G}(\mathrm{d}, \mathrm{p})$ level in chloroform using IEFPCM solvent model were listed in Table 3.

Table 3. The experimental and computed NMR chemical shifts of N-phenylpropanamide.

\begin{tabular}{|c|c|c|c|c|c|c|c|}
\hline Atoms & Exp. $1^{*}$ & Exp. $2^{* * *}$ & Calc. & Atoms & Exp. $1^{*}$ & Exp. $2^{* * *}$ & Calc. \\
\hline $\mathrm{C} 3$ & 139.1 & 138.3 & 148.0 & H12 & 2.34 & 2.35 & 2.54 \\
\hline $\mathrm{C} 4$ & 29.5 & 30.5 & 35.7 & H13 & 2.34 & 2.35 & 2.22 \\
\hline C5 & 171.8 & 173.2 & 178.4 & H14 & 8.41 & 7.90 & 6.95 \\
\hline C6 & 118.9 & 120.4 & 122.5 & H15 & $7.03-7.56$ & 7.50 & 6.96 \\
\hline $\mathrm{C} 7$ & 118.9 & 120.4 & 124.4 & H16 & 7.03-7.56 & 7.50 & 9.13 \\
\hline $\mathrm{C} 8$ & 9.6 & 9.9 & 11.2 & H17 & 1.17 & 1.21 & 0.96 \\
\hline C9 & 128.4 & 128.8 & 135.1 & H18 & 1.17 & 1.21 & 1.02 \\
\hline $\mathrm{C} 10$ & 128.4 & 128.8 & 136.0 & H19 & 1.17 & 1.21 & 1.70 \\
\hline C11 & 122.7 & 124.1 & 129.0 & $\mathrm{H} 20$ & 7.03-7.56 & 7.27 & 7.52 \\
\hline Watar & & & & $\mathrm{H} 21$ & 7.03-7.56 & 7.27 & 7.64 \\
\hline${ }^{{ }^{* *} \text { Taken form AIST }}$ & & & & $\mathrm{H} 22$ & 7.03-7.56 & 7.06 & 7.29 \\
\hline
\end{tabular}

Due to the effect of quite electronegative $\mathrm{O}$ atom, carbons in carbonyl groups resonance in 160-220 ppm region (Lambert et al., 1987; Anderson et al., 2004; Pavia et al., 2009). Due to bonding with the oxygen and nitrogen atoms, NMR chemical shifts for C5 carbon atom was occurred at 171.8/173.2 ppm experimentally and computed at $178.4 \mathrm{ppm}$ as the highest chemical shift in ${ }^{13} \mathrm{C}$ NMR spectrum. From the same reason, the chemical shift of $\mathrm{C} 3$ atom in phenyl ring was observed at $139.1 / 138.3 \mathrm{ppm}$ and calculated at $148.0 \mathrm{ppm}$. Usually, the aromatic ring carbons with $\mathrm{sp}^{2}$ hybrid resonate in the range of 175-100 ppm, depending on the 
substitute groups connected them (Lambert et al., 1987; Anderson et al., 2004; Pavia et al., 2009). The phenyl carbons excepting $\mathrm{C} 3$ in the compound were recorded at the interval of 118129/120-129 ppm and chemical shifts of carbons excepting C3 were calculated in the region of 122-136 ppm. The measured and computed NMR chemical shifts for C4 carbon atom were found at 29.5/30.5 ppm and $35.7 \mathrm{ppm}$, respectively. As expected for methyl carbon, the lowest chemical shift in ${ }^{13} \mathrm{C}$ NMR spectrum was observed for C8 carbon at 9.6/9.9 ppm and calculated at $11.2 \mathrm{ppm}$. Because of the phenyl rings produce broad deshielding effects and $\pi$ bonding electrons behave like a conductor, the hydrogens attached to carbon atoms in aromatic ring give resonance signals in the region of 6-8 ppm (Lambert et al., 1987; Anderson et al., 2004; Pavia et al., 2009). The aromatic protons in title molecule were found at 7.03-7.56/7.06$7.50 \mathrm{ppm}$ as experimental, while they were computed at 6.96-7.64 ppm for the other hydrogens excepting H16 atom. The most deshielded proton (highest chemical shift) H16 atom in the phenyl ring shifted to $9.13 \mathrm{ppm}$ in computations due to the effect of the electronegative $\mathrm{O} 1$ atom. The hydrogen bonded to nitrogen atom in the amide group can give signals in 5-9 ppm region under influence of solvent, concentration and temperature (Pavia et al., 2009). The $\mathrm{H} 14$ atom in $\mathrm{NH}$ group gave NMR signal at 8.41/7.90 ppm, while it was calculated at $6.95 \mathrm{ppm}$. The NMR chemical shifts for protons of methyl and methylene groups were observed at 1.17/1.21 and 2.34/2.35 ppm while they were computed at the interval of 0.96 and $1.70 \mathrm{ppm}$ for methyl protons and 2.22 ppm and $2.44 \mathrm{ppm}$ for methylene protons.

\section{HOMO, LUMO and UV-Vis. Analyses}

FMOs (frontier molecule orbitals), namely, HOMO (highest occupied molecular orbital) and LUMO (lowest unoccupied molecular orbital) are very helpful in interpreting chemical reactions (Fukui, 1982). The HOMOs are donor groups filled by electrons while the LUMOs are acceptor groups unfilled by electrons. They can be used for definition of charge transfer. The HOMOs ( $\mathrm{H}$ and $\mathrm{H}-1)$ and LUMOs (L and $\mathrm{L}+1$ ) plots and their energy values of $\mathrm{N}$ phenylpropanamide were given in Figure 4. HOMO-LUMO energy difference is used as a simple indicator of chemical reactivity. A large HOMO-LUMO gap means high stability and low chemical reactivity (Manolopoulos et al., 1991; Liu et al., 1992). The energy values of HOMO-LUMO band gap was calculated as $5.560 \mathrm{eV}$. As seen from Figure 4, both HOMOs ( $\mathrm{H}$ and $\mathrm{H}-1$ ) and LUMOs ( $\mathrm{L}$ and $\mathrm{L}+1$ ) are mostly formed from bonding and anti-bonding pi molecular orbitals of aromatic ring, respectively. This situation indicates that $\mathrm{H}-1 \rightarrow \mathrm{L}, \mathrm{H}-1 \rightarrow \mathrm{L}+1$, $\mathrm{H} \rightarrow \mathrm{L}$ and $\mathrm{H} \rightarrow \mathrm{L}+1$ transitions are probably $\pi \rightarrow \pi^{*}$ electronic transition.

The computations of UV-Vis. spectral features of $\mathrm{N}$-phenylpropanamide were performed with the TD-DFT/B3LYP/6$311+\mathrm{G}(\mathrm{d}, \mathrm{p})$ level in chloroform by using IEFPCM solvation model. The computed wavelengths, excitation energies, oscillator strengths and major contributions were summarized in Table 4. The major contributions of electronic transitions corresponding to the computed wavelengths were obtained by using GaussSum 3.0 program (O'boyle et al., 2008). The measured (Bio-Rad, 2018) and simulated UV-Vis. spectra in $210-300 \mathrm{~nm}$ region of the molecule were depicted in Figure 5. The strong band observed at $242.0 \mathrm{~nm}$ in the experimental UV-Vis. spectrum of the N-phenylpropanamide can be assigned to $\pi \rightarrow \pi^{*}$ electronic transition that is resulted from delocalize pi electrons of aromatic ring. The computed wavelengths for the three excited states were found at 251.9, 244.0 and $239.5 \mathrm{~nm}$, which are assigned to $\pi \rightarrow \pi^{*}$ transition. Hence, the most intense value computed of oscillator strength was obtained at value of 0.5097 in $\mathrm{H} \rightarrow \mathrm{L}$ electronic transition. 


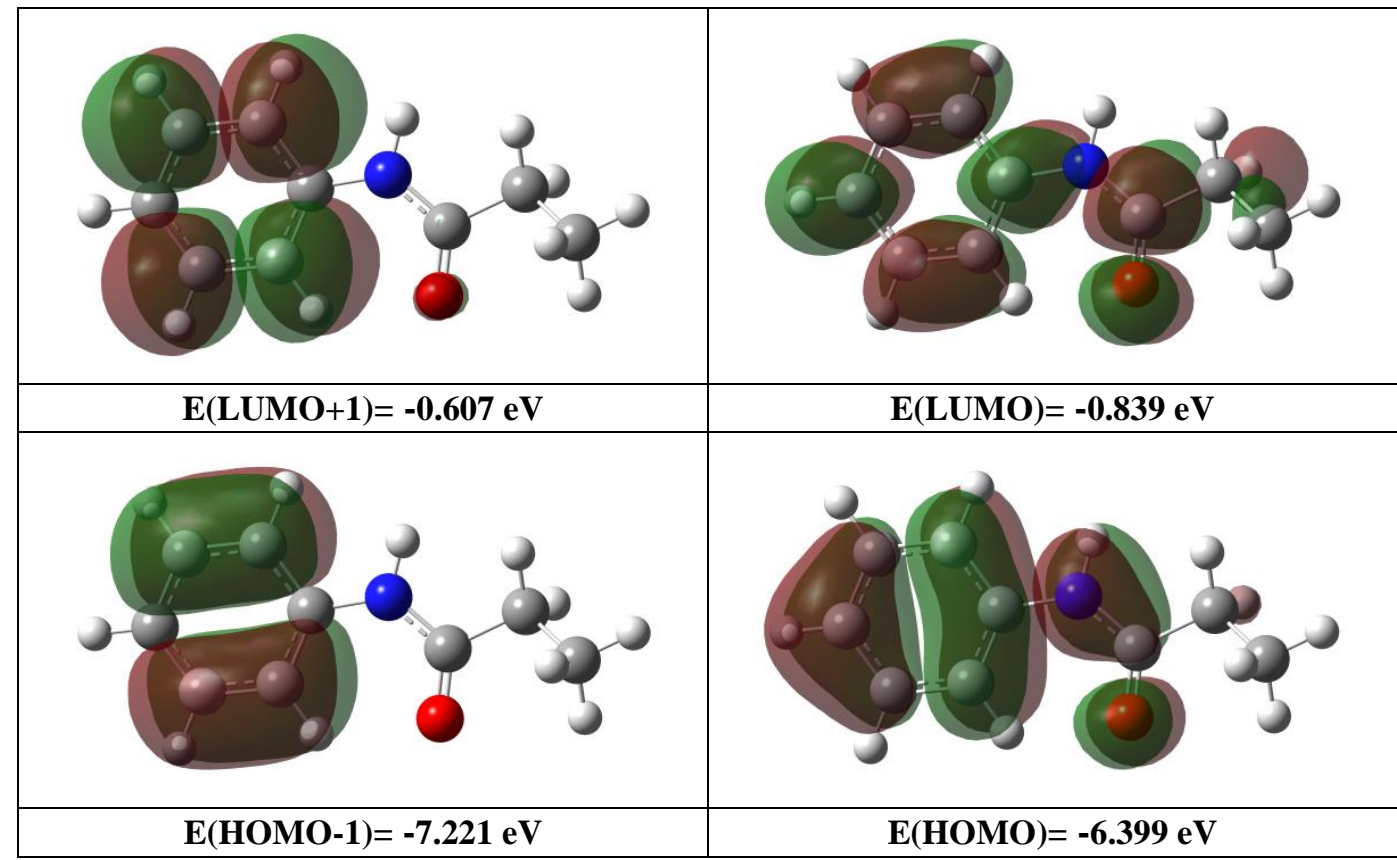

Figure 4. The simulated HOMOs and LUMOs plots of N-phenylpropanamide.

Table 4. The UV-Vis. parameters of N-phenylpropanamide in chloroform.

\begin{tabular}{|c|c|c|c|c|c|}
\hline \multicolumn{2}{|c|}{ Wavelength $(\lambda)(\mathrm{nm})$} & \multirow{2}{*}{ Transition } & \multirow{2}{*}{$\begin{array}{l}\text { Excitation } \\
\text { energy }(\mathrm{eV})\end{array}$} & \multirow{2}{*}{$\begin{array}{c}\text { Oscillator } \\
\text { strengths }(f)\end{array}$} & \multirow{2}{*}{ Major contributions } \\
\hline Exp." & Cal. & & & & \\
\hline \multirow{3}{*}{242.0} & 251.9 & & 4.9224 & 0.0184 & $\mathrm{H} \rightarrow \mathrm{L}+1(74 \%), \mathrm{H}-1 \rightarrow \mathrm{L}(23 \%)$ \\
\hline & 244.0 & $\pi \rightarrow \pi^{*}$ & 5.0806 & 0.5097 & $\mathrm{H} \rightarrow \mathrm{L}(95 \%)$ \\
\hline & 239.5 & & 5.1780 & 0.0005 & $\mathrm{H}-2 \rightarrow \mathrm{L}(88 \%)$ \\
\hline
\end{tabular}

*Taken from Bio-Rad

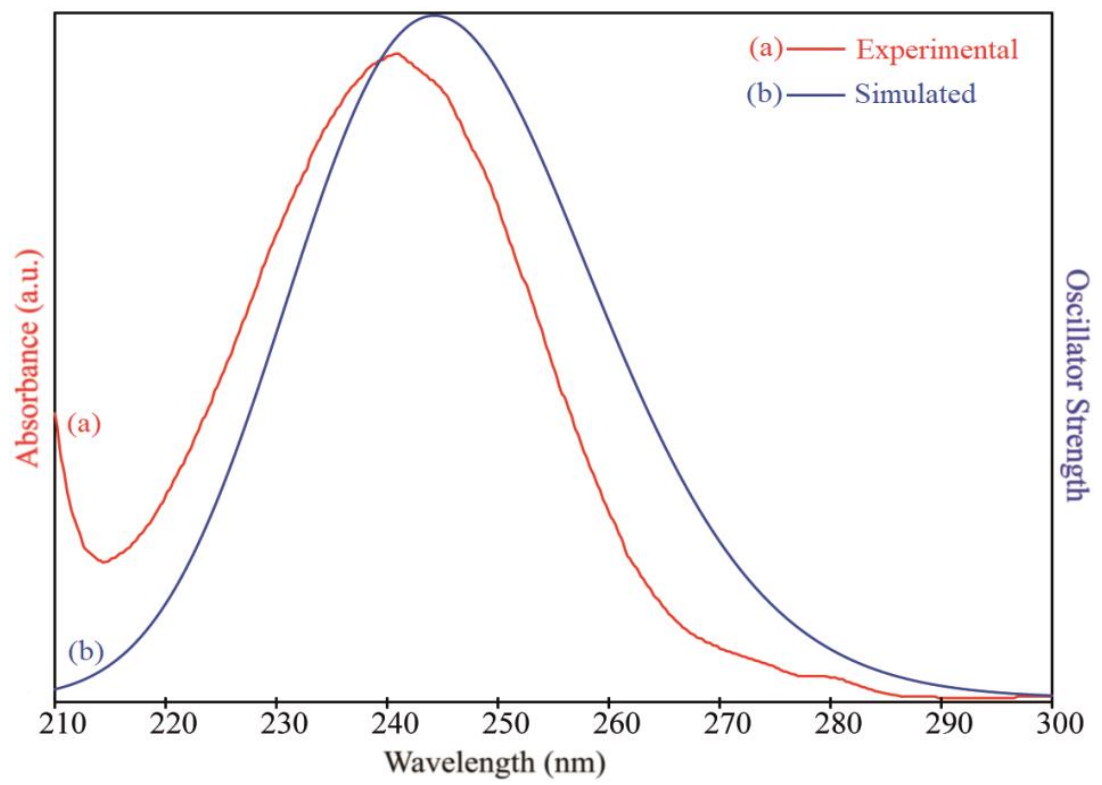

Figure 5. (a) Experimental and (b) simulated UV spectrum of N-phenylpropanamide.

\section{Molecular Electrostatic Potential}

The electrostatic potential of a molecule is a good lead in evaluating the molecular reactivity against positive or negatively charged reagents. The MEP surface has been computed at the B3LYP/6-311+G(d,p) level in gas phase to 
define the electrophilic and nucleophilic sites of the N-phenylpropanamide molecule and it was given in Figure 6. The negative region of the MEP map in gas phase of molecule is mainly localized on the $\mathrm{O}$ atom and the value of electron density is -0.0527 a.u. On the other hand, the positive region is especially localized on the amine hydrogen atom with 0.0586 a.u. value of electron density.

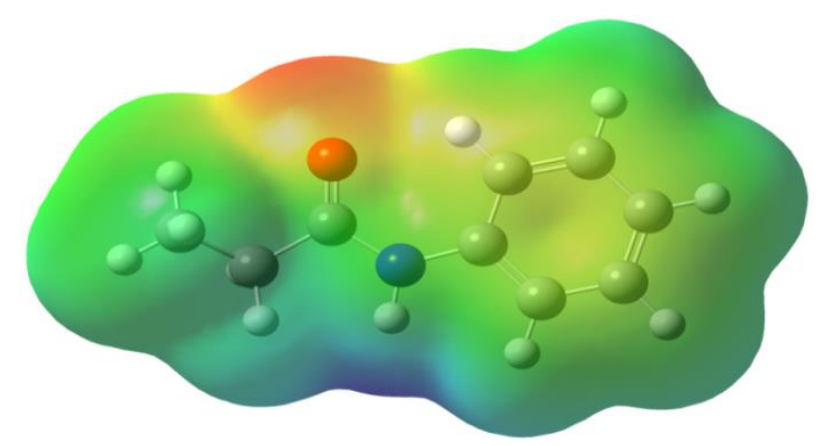

Figure 6. MEP map of N-phenylpropanamide.

\section{CONCLUSION}

The molecular structure, vibrational wavenumbers, proton and carbon-13 NMR isotropic chemical shifts, HOMO-LUMO analyses, UV-Vis. electronic absorption properties and molecular electrostatic potential (MEP) surface of $\mathrm{N}$-phenylpropanamide were examined using experimental and computational methods. The experimental spectroscopic properties taken from literature (vibrational wavenumbers, UV-Vis. wavelengths and NMR chemical shifts) were supported by data computed with the DFT/B3LYP/6-311+G(d,p) level. The $\mathrm{N}-\mathrm{H}, \mathrm{H}^{\cdots} \mathrm{O}$ and $\mathrm{N}^{\cdots} \mathrm{O}$ bond distances and $\mathrm{N}-\mathrm{H}{ }^{\cdots} \mathrm{O}$ bond angle that are the $\mathrm{N}-\mathrm{H}^{\cdots} \mathrm{O}$ intermolecular hydrogen bond parameters were obtained as $1.016 \AA, 2.020 \AA, 3.034 \AA$ and $175.0^{\circ}$ respectively. Similarly, the $\mathrm{NH}$ and $\mathrm{C}=\mathrm{O}$ stretching frequencies were computed at 3374 $\mathrm{cm}^{-1}$ and $1652 \mathrm{~cm}^{-1}$ due to the $\mathrm{N}-\mathrm{H}^{\cdots} \mathrm{O}$ intermolecular interaction. Due to bonding electronegative atoms, NMR chemical shifts for C3 and C5 carbon atom was occurred at 171.8/173.2 ppm and 139.1/138.3 ppm experimentally and computed at $148.0 \mathrm{ppm}$ and $178.4 \mathrm{ppm}$ as the highest chemical shift in ${ }^{13} \mathrm{C}$ NMR spectrum. The electronic transitions were confirmed with theoretical HOMO, LUMO and $\mathrm{UV}-\mathrm{Vis}$. analyses of the title molecule. Because of the HOMOs and LUMOs are mostly formed from bonding and anti-bonding pi molecular orbitals of aromatic ring, respectively, transitions were explicated as $\pi \rightarrow \pi^{*}$ electronic transition.

\section{REFERENCES}

AIST, 2018. Spectral Database for Organic Compounds, SDBS. http://sdbs.db.aist.go.jp (Date of Access: 30 October 2018).

Anderson MP, Uvdal P, 2005. New Scale Factors for Harmonic Vibrational Frequencies Using the B3LYP Density Functional Method with the Triple- $\zeta$ Basis Set $6-311+\mathrm{G}(\mathrm{d}, \mathrm{p})$. The Journal of Physical Chemistry A, 109: 29372941.

Anderson RJ, Bendell DJ, Groundwater PW, 2004. Organic Spectroscopic Analysis. The Royal Society of Chemistry, Sanderland-UK.

Becke AD, 1993. Density-Functional Thermochemistry. III. The Role of Exact Exchange. The Journal of Chemical Physics, 98: 5648-5652.

Bellamy LJ, 1975. The Infrared Spectra of Complex Molecules. Wiley, New YorkUSA.

Bilkan MT, 2019. Quantum chemical studies on solvent effects, ligand-water complexes and dimer structure of 2,2'-dipyridylamine. Physics and Chemistry of Liquids, 57(1): 100-116. 
Bilkan MT, 2017. Structural and spectroscopic studies on dimerization and solvent-ligand complexes of Theobromine. Journal of Molecular Liquids, 238: 523-532.

Bio-Rad Laboratories, 2018. Inc. SpectraBase. http://spectrabase.com/ad?a=SPECTRUM_J Wm7vAp51SI\&r (Date of Access: 30 October 2018).

Colthup NB, Daly LH, Wiberley E, 1964. Introduction to Infrared and Raman Spectroscopy. Academic Press, New YorkUSA.

Dennington R, Keith T, Millam J, 2009. GaussView, Version 5, Semichem Inc., Shawnee Mission KS.

Diab MA, El-Sonbati AZ, El-Bindary AA, Abd ElGhany HM, 2017. Thermal Stability and Degradation of Poly $(\mathrm{N}$ phenylpropionamide) Homopolymer and Copolymer of N-Phenylpropionamide with Methyl Methacrylate. Arabian Journal of Chemistry, 10(2): S3732-S3739.

Ditchfield R, 1974. Self-Consistent Perturbation

Theory of Diamagnetism. Molecular Physics, 27(4): 789-807.

Edreva AM, Velikova VB, Tsonev TD, 2007. Phenylamides in Plants. Russian Journal of Plant Physiology, 54(3): 287-301.

Frisch MJ, Trucks GW, Schlegel HB, Scuseria GE, Robb MA, Cheeseman JR, Scalmani G, Barone V, Mennucci B, Petersson GA, Nakatsuji H, Caricato M, Li X, Hratchian $\mathrm{HP}$, Izmaylov AF, Bloino $\mathrm{J}$, Zheng $\mathrm{G}$, Sonnenberg JL, Hada M, Ehara M, Toyota K, Fukuda R, Hasegawa J, Ishida M, Nakajima T, Honda Y, Kitao O, Nakai H, Vreven T, Montgomery JA Jr., Peralta JE, Ogliaro F, Bearpark M, Heyd JJ, Brothers E, Kudin KN, Staroverov VN, Kobayashi R, Normand J, Raghavachari K, Rendell A, Burant JC, Iyengar SS, Tomasi J, Cossi M, Rega N, Millam JM, Klene M, Knox JE, Cross JB, Bakken V, Adamo C, Jaramillo J, Gomperts R, Stratmann RE, Yazyev O, Austin A.J, Cammi R, Pomelli C, Ochterski JW, Martin RL, Morokuma K, Zakrzewski
VG, Voth GA, Salvador P, Dannenberg JJ, Dapprich S, Daniels AD, Farkas O, Foresman JB, Ortiz JV, Cioslowski J, Fox DJ, 2009. Gaussian 09, Revision A.1, Gaussian, Inc., Wallingford CT-USA.

Fukui K, 1982. The Role of Frontier Orbitals in Chemical Reactions (Nobel Lecture). Angewandte Chemie, 21(11): 801-809.

Jamr'oz MH, 2004. Vibrational Energy Distribution Analysis VEDA4, WarsawPoland.

Klees TM, Sheffels P, Thummel KE, Kharasch ED, 2005. Pharmacogenetic Determinants of Human Liver Microsomal Alfentanil Metabolism and the Role of Cytochrome P450 3A5, Anesthesiology, 102(3): 550-556. Lambert JB, Shurvell HF, Cooks RG, 1987. Introduction to Organic Spectroscopy. Macmillan Publishing, New York-USA.

Lee C, Yang W, Parr RG, 1988. Development of the Colle-Salvetti Correlation-Energy Formula Into a Functional of the Electron Density. Physical Review B, 37: 785-789.

Liu L-F. Liu H, Pi H-J, Yang S, Yao M, Du W, Deng W-P, 2011. Facile $\mathrm{AlCl}_{3}$-Promoted Catalytic Beckmann Rearrangement of Ketoximes. Synthetic Communications: An International Journal for Rapid Communication of Synthetic Organic Chemistry, 41(4): 553-560.

Liu X, Schmalz TG, Klein DJ, 1992. Favorable Structures for Higher Fullerenes. Chemical Physics Letters, 188(5-6): 550-554.

London F, 1937. Théorie Quantique Des Courants Interatomiques Dans Les Combinaisons Aromatiques. Journal of Physical Radium, 8(10): 397-409.

Manolopoulos DE, May JC, Down SE, 1991. Theoretical Studies of the Fullerenes: $\mathrm{C}_{34}$ to $\mathrm{C}_{70}$. Chemical Physics Letters, 181(2-3): 105-111. 
Mierina I, Gudelis E, Stepanovs D, Jure M, Mishnev A, Kolympadi M, Marković D, 2016. Crystal structure of 3-(4-hydroxy-3methoxyphenyl)-N-phenylpropanamide, $\mathrm{C}_{16} \mathrm{H}_{17} \mathrm{NO}_{3}$. Zeitschrift für Kristallographie New Crystal Structures, 231(2): 657-659.

Miertus S, Scrocco E, Tomasi J, 1981. Electrostatic Interaction of a Solute with a Continuum. A Direct Utilizaion of AB Initio Molecular Potentials for the Prevision of Solvent Effects. Chemical Physics, 55(1): 117-129.

Na A, Hongjun P, Lifeng L, Wenting D, Weiping D, 2011. A Mild and Highly Efficient Catalyst for Beckmann Rearrangement, $\mathrm{BF}_{3} \bullet \mathrm{OEt}_{2}$. Chinese Journal of Chemistry, 29(5): 947-950.

Nowrouzi N, Jonaghani MZ, 2012. Highly Selective Mono-N-benzylation and Amidation of Amines with Alcohols or Carboxylic Acids Using the $\mathrm{Ph}_{2} \mathrm{PCl} / \mathrm{I}_{2} /$ imidazole Reagent System. Canadian Journal of Chemistry, 90(60): 498509.

O'boyle NM, Tenderholt AL, Langner KM, 2008. Cclib: A Library for Package-Independent Computational Chemistry Algorithms. Journal of Computational Chemistry, 29, 839-845.

Öztürk N, Özdemir T, Alpaslan YB, Gokce H, Alpaslan G, 2018. Experimental (FT-IR, Raman and NMR) and Theoretical (B3LYP, B3PW91, M06-2X and CAM-B3LYP) Analyses of P-Tert-Butylphenyl Salicylate. Bilge International Journal of Science and Technology Research, 2(1): 56-73.
Pavia DL, Lampman GM, Kriz GS, Vyvyan JR, 2009. Introduction to Spectroscopy. Brooks/Cole Cengage Learning, USA.

Priyadharsini P, Dhanasekaran D, Kanimozhi B, 2013. Isolation, Structural Identification and Herbicidal Activity of N-phenylpropanamide from Streptomyces sp. $\mathrm{KA}_{1}-3$, Archives Of Phytopathology And Plant Protection, 46(3): 364-373.

Runge E, Gross EKU, 1984. Density-Functional Theory for Time-Dependent Systems. Physical Review Letters, 52(12): 997-1000.

Silverstein RM, Webster FX, 1998. Spectroscopic Identification of Organic Compounds. $6^{\text {th }}$ ed., John Wiley \& Sons, New York-USA.

Stuart BH, 2004. Infrared Spectroscopy: Fundamentals and Applications. JohnWilley $\&$ Sons, England.

Watanabe Y, Tsuji Y, Kondo T, Takeuchi R, 1984. Platinum Complex Catalyzed Reductive NAcylation of Nitro Compounds. The Journal Of Organic Chemistry, 1984, 49(23): 44514455.

Wolinski K, Hinton JF, Pulay P, 1990. Efficient Implementation of the Gauge-Independent Atomic Orbital Method for NMR Chemical Shift Calculations. Journal of the American Chemical Society, 112(d): 8251-8260.

Yıldırım MH, 2018. Infrared and NMR Spectral Analyses and Computational Studies of 2amino-3-methylbenzoic acid. Bilge International Journal of Science and Technology Research, 2(1): 74-82. 